\title{
INSTABILITY AND THEORIES WITH FEW MODELS
}

\author{
A. PILlay ${ }^{1}$
}

\begin{abstract}
Some results are obtained concerning $n(T)$, the number of countable models up to isomorphism, of a countable complete first order theory $T$. It is first proved that if $n(T)=3$ and $T$ has a tight prime model, then $T$ is unstable. Secondly, it is proved that if $n(T)$ is finite and more than one, and $T$ has few links, then $T$ is unstable. Lastly we show that if $T$ has an algebraic model and has few links, then $n(T)$ is infinite.
\end{abstract}

We work with countable complete theories. Under certain assumptions which simplify the structure of a theory, we prove that such a theory with few countable models (finitely many and more than one, or in some cases exactly three) is unstable. The first condition is that every countable model of $T$ has an elementary substructure which is prime and contains every other prime elementary substructure. This condition is first examined and related to other properties of prime models. Then it is shown that if such a theory has exactly three countable models then it is unstable. Secondly we look at the condition that a theory has 'few links'. This was introduced by Benda in [1], and roughly speaking says that given any two types there are few ways of relating them in a nonprincipal way. Benda proved that a theory with few links and few countable models has at least two countable universal models. We show that a theory with few links and a finite number of countable models is unstable. (A weaker property than few links is actually needed.) Actually we show that such a theory 'says' that there is a dense ordering, and we conclude that a theory with few links and an algebraic model has infinitely many countable models.

1. Tight prime models. We assume here that $T$ is atomic (the principal types are dense in the space of (complete) types). We know that such a theory has a prime model (elementarily embeddable in all models of $T$ ) which is unique up to isomorphism.

Definition 1. Let $M$ be the prime model of $T$. Then $M$ is said to be tight if for any countable model $N$ of $T$, the set of elementary substructures of $N$ which are isomorphic to $M$, has a greatest element under the ordering by inclusion.

We recall that a minimal model is a model with no proper elementary substructure. If $T$ has a minimal model then it is unique and prime.

Received by the editors April 12, 1979.

1980 Mathematics Subject Classification. Primary 03C15, 03C45; Secondary 03 C50.

Key words and phrases. Unstable theory, the order property, tight prime model, theory with few links, algebraic model.

'This research was undertaken while the author was the recipient of a Royal Society European Science Exchange Fellowship. 
Recall also that a model $M$ is algebraic, if every element of $M$ is contained in a finite definable subset of $M$ (definable without parameters). An algebraic model is clearly minimal and prime.

There are prime models which are tight but not minimal. An example is the countable model of an $\aleph_{0}$-categorical theory. Another is the prime model of the Ehrenfeucht example, i.e. the ordered rationals with the natural numbers named by constants. There are also prime minimal models which are not tight, for example the prime model of the theory of discrete linear orderings.

The following is easy to prove.

Proposition 2. Let $M$ be prime. Then $M$ is algebraic if and only if $M$ is tight and minimal.

We have the following characterisation of theories with tight models.

Proposition 3. $T$ has a tight prime model if and only if whenever $\bar{a}$ and $\bar{b}$ are tuples in a model of $T$, each realising a principal type, then the tuples $\bar{a}^{n} \bar{b}$ realises $a$ principal type.

Proof. Let us suppose that $T$ has a tight prime model $M$. Let $\bar{a}$ and $\bar{b}$ realise principal types in a model $N$ of $T$. We may suppose that $N$ is countable. Let $\bar{a}^{\prime}$ and $\bar{b}^{\prime}$ realise the types of $\bar{a}$ and $\bar{b}$ respectively in $M$ (as these types are principal). Then by the classical argument there are elementary embeddings $f$ and $g$ of $M$ in $N$ such that $f\left(\bar{a}^{\prime}\right)=\bar{a}$, and $g\left(\bar{b}^{\prime}\right)=\bar{b}$. As $M$ is tight there is $M^{\prime} \prec N$ with $M^{\prime} \simeq M$ such that every prime elementary substructure of $N$ is included in $M^{\prime}$. It follows that $\bar{a}$ and $\bar{b}$ are inside $M^{\prime}$. But $M^{\prime}$ is atomic, so $\bar{a}^{\wedge} \bar{b}$ realises a principal type in $M^{\prime}$ and so also in $N$.

Conversely, suppose that the right-hand side is satisfied. Let $N$ be any countable model of $T$. Let $M$ be the set of elements of $N$ which realise principal 1-types in $N$. We first show that $M$ is an elementary substructure of $N$, by the Tarski criterion. So suppose that $\bar{a}$ is a tuple of elements of $M$ and $N \vDash \operatorname{Ex} \varphi(x, \bar{a})$. Now it follows from the condition on the right-hand side that the tuple $\bar{a}$ realises a principal type in $N$. So it is easy to see that as $T$ is atomic, so is $T^{\prime}=\operatorname{Th}(N, \bar{a})$. So let $\psi(x, \bar{a})$ be a complete 1-formula of $T^{\prime}$ which implies $\varphi(x, \bar{a})$. Let $b$ be in $N$ such that $N$ F $\psi(b, \bar{a})$. Then $b$ is principal over $\bar{a}$ in $N$, and as the type of $\bar{a}$ is principal, so is the type of $b$. Then $b \in M$, and as clearly $N \vDash \varphi(b, \bar{a})$ we are finished. Again it follows from our condition, that every tuple in $M$ realises a principal type. Thus $M$ is prime. But if $M^{\prime}$ is some elementary substructure of $N$ which is also prime, then every element of $M^{\prime}$ realises a principal 1-type in $N$, and so $M^{\prime}$ is included in $M$. Thus $T$ has a tight prime model.

2. Theories with three models. In this section we prove the following theorem.

THEOREM 4. Suppose that $T$ has exactly three countable models, and that its prime model is tight. Then $T$ is unstable.

We will show that $T$ has the order property. This says (Shelah [2]) that there is a formula $\psi(\bar{x}, \bar{y})$ and tuples $\bar{a}_{i}$ for $i<\omega$, of the same length as $\bar{y}$, in some model of 
$T$, such that for each $r<\omega$ the set of formulae

$$
p_{r}=\left\{\psi\left(\bar{x}, \bar{a}_{i}\right): i>r\right\} \cup\left\{\neg \psi\left(\bar{x}, \bar{a}_{i}\right): i<r\right\} \quad \text { is consistent. }
$$

As much of the argument is given in detail in $[3, \S \S 1$ and 2$]$, we just sketch the main steps of the first part of the proof, as a series of facts. Let us first mention that in all the known examples of theories with three countable models, the prime model is tight.

FACT (i). Suppose that $M$ is a model of $T, M$ is prime over $\bar{c}$, where type $(\bar{c})=p$ is nonprincipal, and $M$ realises all types of $T$. Then there is $\bar{d}$ in $M$, whose type is also $p$, such that $\bar{c}$ is not principal over $\bar{d}$.

FACT (ii). Suppose that $T$ has exactly three countable models. Then there is a model $M$ of $T$ which realises all types of $T$, such that for every nonprincipal type $p$ of $T$ there is $\bar{c}$ in $M$ such that type $(\bar{c})=p$ and $M$ is prime over $\bar{c}$. (By $M$ being prime over $\bar{c}$ we mean that $(M, \bar{c})$ is a prime model of its theory.)

FACT (iii). Suppose that $T$ is not $\aleph_{0}$-categorical, and that $T$ has $\aleph_{0}$ types. Then for some $n<\omega$ there is an $n$-type $p$ of $T$ of Cantor-Bendixon rank 1 , i.e. there is an $n$-formula $\theta(\bar{x})$ such that $p$ is determined over $T$ by the set of formulae

$$
\{\theta(\bar{x})\} \cup\{\neg \varphi(\bar{x}): \varphi \in \Phi\}
$$

where $\Phi$ is the set of those complete $n$-formulae (generators of principal types) $\varphi(\bar{x})$ such that $T \vdash \varphi \rightarrow \theta$.

As our $T$ has three countable models, Fact (iii) applies. So by (i) and (ii) we assume from now on that

Assumption (iv). $p$ is an $n$-type of $T$ of Cantor-Bendixon rank 1 (determined by formulae as in (iii)), $M$ is a model of $T$ prime over $\bar{c}$ where the type of $\bar{c}$ is $p$, and there is $\bar{d}$ in $M$ with type $p$ such that $\bar{c}$ is not principal over $\bar{d}$.

As $\bar{d}$ is clearly principal over $\bar{c}$, let $\psi(\bar{x}, \bar{y})$ be a formula such that $M \vDash \psi(\bar{c}, \bar{d})$ and $\psi(\bar{c}, \bar{y})$ is a complete $n$-formula of $\operatorname{Th}(M, \bar{c})$.

FACT (v). As $\psi(\bar{c}, \bar{y})$ is complete and $\bar{c}$ is not principal over $\bar{d}$, it follows that there are infinitely many formulae $\varphi \in \Phi$ such that

$$
M \vDash E \bar{x}(\varphi(\bar{x}) \wedge \psi(\bar{x}, \bar{c})) .
$$

FACT (vi). Notice that $T \cup p(\bar{x}) \vdash \psi(\bar{x}, \bar{y}) \rightarrow p(\bar{y})$. Applying compactness and using (v), it follows that there are $\varphi_{i} \in \Phi$ for each $i<\omega$ such that

(I) if $i<j$, then $M \vDash \neg E \bar{y} E \bar{x}\left(\varphi_{j}(\bar{y}) \wedge \varphi_{i}(\bar{x}) \wedge \psi(\bar{y}, \bar{x})\right)$ and

(II) for all $i<\omega M \vDash E \bar{x}\left(\varphi_{i}(\bar{x}) \wedge \psi(\bar{x}, \bar{c})\right)$.

We now show that the formula $\psi$ has the order property. We define by induction on $r<\omega$, natural numbers $r_{1}, r_{2}$, and $n$-tuples $\bar{c}_{r}$ and $\bar{a}_{r}$ from $M$, such that

(A) $r_{1}<r_{2}$, and $s_{1}, s_{2}<r_{1}$ for $s<r$.

(B) $M \vDash \varphi_{r_{1}}\left(\bar{c}_{r}\right)$ and $M \vDash \psi\left(\bar{c}_{r}, \bar{c}\right)$.

(C) $M \vDash \varphi_{r_{2}}\left(\bar{a}_{r}\right)$ and $M \vDash \psi\left(\bar{c}_{s}, \bar{a}_{r}\right)$ for $s<r$.

So suppose that we have defined $r_{1}, r_{2}, \bar{c}_{r}, \bar{a}_{r}$, for $r<m$ satisfying (A), (B) and (C). We will now define them for $r=m$.

First let $m_{1}=(m-1)_{2}+1$. By Fact (vi)(II), we can pick $\bar{c}_{m}$ such that $M$ F $\varphi_{m_{1}}\left(\bar{c}_{m}\right) \wedge \psi\left(\bar{c}_{m}, \bar{c}\right)$. Thus (B) is satisfied. 
Now as for each $r<m$ we have $M \vDash \varphi_{r_{1}}\left(\bar{c}_{r}\right)$, and as each $\varphi_{r_{1}}$ is a complete $n$-formula of $T$, each $\bar{c}_{r}$ therefore realises a principal type in $M$. Thus from the hypothesis that $T$ has a tight prime model and by Proposition 3, it follows that the tuple $\bar{c}_{0} \cap \bar{c}_{1} \cap \ldots \cap \bar{c}_{m}$ realises a principal type in $M$. So let $\sigma\left(\bar{x}_{0}, \bar{x}_{1}, \ldots, \bar{x}_{m}\right)$ be a complete formula of $T$ generating this principal type. As $M \vDash \psi\left(\bar{c}_{r}, \bar{c}\right)$ for every $c<m$, we have

$$
M \vDash E \bar{x}_{0} \bar{x}_{1} \ldots \bar{x}_{m}\left(\sigma\left(\bar{x}_{0}, \bar{x}_{1}, \ldots, \bar{x}_{m}\right) \wedge \psi\left(\bar{x}_{0}, \bar{c}\right) \wedge \psi\left(\bar{x}_{1}, \bar{c}\right) \wedge \ldots \wedge \psi\left(\bar{x}_{m}, \bar{c}\right)\right) .
$$

Let the sentence above be denoted by $\chi(\bar{c})$. Then as the type of $\bar{c}$ is $p$, we have $T \cup p(\bar{y}) \vdash \chi(\bar{x})$. Applying compactness, there is a finite subset $\Phi^{\prime}$ of $\Phi$ such that $T \cup\{\theta(\bar{y})\} \cup\left\{\neg \varphi(\bar{y}): \varphi \in \Phi^{\prime}\right\} \vdash \chi(\bar{y})$. As $\Phi^{\prime}$ is finite we can choose $m_{2}>m_{1}$ such that $\varphi_{m_{2}} \notin \Phi^{\prime}$. As $\varphi_{m_{2}} \in \Phi$ we have that $T \vdash \varphi_{m_{2}}(\bar{y}) \rightarrow \theta(\bar{y})$. So $T \vdash \forall \bar{y}\left(\varphi_{m_{2}}(\bar{y})\right.$ $\rightarrow \chi(\bar{y}))$. It trivially follows that

$$
T \vdash E \bar{x}_{0} \bar{x}_{1} \ldots \bar{x}_{m}\left(\sigma\left(\bar{x}_{0}, \ldots, \bar{x}_{m}\right) \wedge E \bar{y}\left(\varphi_{m_{2}}(\bar{y}) \wedge \psi\left(\bar{x}_{0}, \bar{y}\right) \wedge \ldots \wedge \psi\left(\bar{x}_{m}, \bar{y}\right)\right)\right) .
$$

Then by the completeness of the formula $\sigma\left(\bar{x}_{0}, \ldots, \bar{x}_{m}\right)$ we have

$$
T \vdash \forall \bar{x}_{0} \ldots \bar{x}_{m}\left(\sigma\left(\bar{x}_{0}, \ldots, \bar{x}_{m}\right) \rightarrow E \bar{y}\left(\varphi_{m_{2}}(\bar{y}) \wedge \psi\left(\bar{x}_{0}, \bar{y}\right) \wedge \ldots \wedge \psi\left(\bar{x}_{m}, \bar{y}\right)\right)\right) .
$$

Note that $M \vDash \sigma\left(\bar{c}_{0}, \ldots, \bar{c}_{M}\right)$. Thus we can pick $\bar{a}_{m}$ in $M$ such that $M \vDash \varphi_{m_{2}}\left(\bar{a}_{m}\right) \wedge$ $\psi\left(\bar{c}_{0}, \bar{a}_{m}\right) \wedge \ldots \wedge \psi\left(\bar{c}_{m}, \bar{a}_{m}\right)$. Then (A), (B) and (C) are clearly satisfied for $r=m$, and the induction step is finished. So we have $\bar{c}_{r}$ and $\bar{a}_{r}$ defined for all $r<\omega$, such that if $i>r$ then $M \vDash \psi\left(\bar{c}_{r}, \bar{a}_{i}\right)$. If $i<r$ then we have by (A) that $i_{2}<r_{1}$, and thus by Fact (vi)(I) we have $M \vDash \neg \psi\left(\bar{c}_{r}, \bar{a}_{i}\right)$. Thus for each $r<\omega \bar{c}_{r}$ satisfies the type $p_{r}$ defined at the beginning of this section. So each $p_{r}$ is consistent, whereby $\psi$ has the order property, and by [2] $T$ is unstable.

\section{Theories with few links.}

Definition (BENDA). $T$ is said to have few links if whenever $p(\bar{x})$ and $q(\bar{y})$ are types of $T$ (with $\bar{x}$ and $\bar{y}$ disjoint sets of variables), then there are only a finite number of types $r(\bar{x}, \bar{y}) \supseteq p(\bar{x}) \cup q(\bar{y})$ such that $r(\bar{c}, \bar{y})$ is a nonprincipal type of $T \cup p(\bar{c})$ (where $\bar{c}$ are new constants).

This condition is admittedly rather artificial, but it enables some proofs to go through. In fact all we will need is that the above property hold in the case that $p$ and $q$ are the same type.

THEOREM 5. If $T$ has finitely many (and more than one) countable models, and few links, then $T$ is unstable.

Proof. The idea is to show that there is a definable ordering on an infinite set. Under the assumption of few links, this is almost trivial. However we then go on (under the same assumptions of few links and few models) to show that we can assume the ordering to be dense, and then we draw some further conclusions. So until we say otherwise, we assume

Assumption 6. $T$ has finitely many but more than one countable model, and has few links.

We know immediately that $T$ has a model, $M$ say, which realises all types of $T$, and is prime over a finite set (e.g. Benda [1]). We suppose that $M$ is prime over $\bar{c}$, 
and that the type of $\bar{c}$ is $p \cdot p$ must be nonprincipal, otherwise $T$ is $\aleph_{0}$-categorical. So, by Fact (i) of the last section, there is $\bar{d}$ in $M$ with type $p$, such that $\bar{c}$ is not principal over $\bar{d}$. Now any tuple of $M$ is principal over $\bar{c}$, so realises a complete formula over $\bar{c}$. So let $\Psi$ be the set of formulae $\psi(\bar{x}, \bar{y})$ such that $\psi(\bar{c}, \bar{y})$ is a complete formula of $\operatorname{Th}(M, \bar{c})$, and there is $\bar{d}$ in $M$ realising $p, \bar{c}$ is nonprincipal over $\bar{d}$, and $M \vDash \psi(\bar{c}, \bar{d})$. Clearly for each $\psi \in \Psi, M \vDash \psi(\bar{c}, \bar{x}) \rightarrow p(\bar{x})$. Also each $\psi$ clearly corresponds to a nonprincipal link of $p$ and $p$. Thus, by the assumption of few links, $\Psi$ is finite. So $\bigvee \Psi(\bar{x}, \bar{y})$ is a first order formula (i.e. finitary). Denote $\bigvee \Psi(\bar{x}, \bar{y})$ by $\varphi(\bar{x}, \bar{y})$.

LEMMA 7. It is true that $M \vDash \forall \bar{x} \bar{y}(\varphi(\bar{c}, \bar{x}) \wedge \varphi(\bar{x}, \bar{y}) \rightarrow \varphi(\bar{c}, \bar{y}))$.

Proof. So let $M \vDash \varphi(\bar{c}, \bar{d}) \wedge \varphi(\bar{d}, \bar{e})$ for some $\bar{d}, \bar{e}$ in $M$. Then the type of $\bar{d}$ and thus of $\bar{e}$ is also $p$. As $M$ is prime over $\bar{c}, \bar{e}$ is principal over $\bar{c}$. Thus to show that $M \vDash \varphi(\bar{c}, \bar{e})$ it is enough to show that $\bar{c}$ is not principal over $\bar{e}$. If $\bar{c}$ was principal over $\bar{e}$, then there would be a formula $\sigma_{1}(\bar{e}, \bar{x})$ realised by $\bar{c}$ such that $M \vDash \sigma_{1}(\bar{e}, \bar{x})$ $\rightarrow p(\bar{x})$. Note that $\bar{e}$ is principal over $\bar{d}$. Thus there would be a formula $\sigma_{2}(\bar{d}, \bar{x})$ satisfied by $\bar{c}$, such that $M \vDash \sigma_{2}(\bar{d}, \bar{x}) \rightarrow p(\bar{x})$. This, together with the fact that $\bar{d}$ is principal over $\bar{c}$, can be shown to imply that $\bar{c}$ is principal over $\bar{d}$. But this contradicts $M \vDash \varphi(\bar{c}, \bar{d})$. Thus $\bar{c}$ is not principal over $\bar{e}$, and we are finished.

It is now simple to see that the formula $\varphi(\bar{x}, \bar{y})$ defines a total ordering on an infinite set of tuples. For let $\bar{c}_{i}, i<\omega$, be defined in $M$ such that $\bar{c}_{0}=\bar{c}$, and $M \vDash \varphi\left(\bar{c}_{i}, \bar{c}_{i+1}\right)$. We can carry on the construction, as every $\bar{c}_{i}$ has the same type as $\bar{c}$. Lemma 7, for the same reason gives us transitivity. As clearly $M \vDash \varphi(\bar{c}, \bar{x}) \rightarrow \bar{c} \neq$ $\bar{x}$, we also have irreflexivity. Thus $T$ is unstable, and the proof of Theorem 5 is complete.

However, now we continue the argument, still working under Assumption 6. We would like to show that the ordering which $\bigvee \Psi$ defines is dense. This is not so easy, so we show

LEMMA 8. There is a nonempty $\Phi \subseteq \Psi$, such that $M \vDash \forall \bar{x} \bar{y}(\bigvee \Phi(\bar{c}, \bar{x}) \wedge$ $\bigvee \Phi(\bar{x}, \bar{y}) \rightarrow \bigvee \Phi(\bar{c}, \bar{y}))$ and $M \vDash \forall \bar{x}(\bigvee \Phi(\bar{c}, \bar{x}) \rightarrow E \bar{y}(\bigvee \Phi(\bar{c}, \bar{y}) \wedge \bigvee \Phi(\bar{y}, \bar{x})))$

Proof. We describe a tree $T$ which has three levels. Level 0 consists just of $\bar{c}$. Level 1 consists of those $\bar{x} \in M$ such that there is $\psi \in \Psi$ and $M \vDash \psi(\bar{c}, \bar{x})$. We write Level 1 as $\left\{\bar{d}_{i}: i \in I\right\}$. Level 2 is enumerated as $\bar{e}_{i, j}$, where for each $i \in I,\left\{\bar{e}_{i, j}: j \in i_{J}\right\}$ is the set of those $\bar{x}$ in $M$ such that $M \vDash \psi\left(\bar{d}_{\underline{i}}, \bar{x}\right)$ for some $\psi \in \Psi$. Note that if $\psi \neq \psi^{\prime}$, and $M \vDash \psi\left(\bar{c}, \bar{d}_{i}\right) \wedge \psi^{\prime}\left(\bar{c}, \bar{d}_{i^{\prime}}\right)$, then $\bar{d}_{i} \neq \bar{d}_{i^{\prime}}$, as each $\psi$ is a complete formula. The same is true for the elements above any $\bar{d}_{i}$, as $\bar{d}_{i}$ has the same type as $\bar{c}$. For the same reason, every $\psi$ is represented among the $\bar{e}_{i, j}$ for each $i$. Notice that it is possible that there are $i \neq i^{\prime}$, and $j, j^{\prime}$ such that $\bar{e}_{i, j}$ is the same tuple as $e_{i^{\prime}, j^{\prime}}$. However we still treat $\bar{e}_{i, j}$ and $\bar{e}_{i^{\prime}, j^{\prime}}$ as distinct members of the tree.

By Lemma 7, for each $\bar{e}$ in Level 2, there is $\psi \in \Psi$ such that $M \vDash \psi(\bar{c}, \bar{e})$. Let $\Psi^{(1)}$ be the subset of $\Psi$ consisting of those $\psi \in \Psi$ such that there is $\bar{e}$ in Level 2, such that $M \vDash \psi(\bar{c}, \bar{e})$. So $\Psi^{(1)}$ is nonempty. Let now $T^{(1)}$ be the subtree of the above tree $T$ described as follows. $\bar{c}$ is still Level 0 of $T^{(1)}$. Level 1 consists of those $\bar{d}_{i}$ such 
that there is $\psi \in \Psi^{(1)}$ with $M \vDash \psi\left(\bar{c}, \bar{d}_{i}\right)$. For Level 2, $\bar{e}_{i, j}$ is in $T^{(1)}$ iff $\bar{d}_{i}$ is in $T^{(1)}$ and there is $\psi \in \Psi^{(1)}$ such that $M \vDash \psi\left(\bar{d}_{i}, \bar{e}_{i, j}\right)$. It is clear that there are elements at Level 2 of $T^{(1)}$. Thus we can define $\Psi^{(2)}$ as the subset of $\Psi^{(1)}$ consisting of those $\psi$ such that there is $\bar{e}$ in Level 2 of $T^{(1)}$ with $M \vDash \psi(\bar{c}, \bar{e})$. Again we can define a subtree $T^{(2)}$ of $T^{(1)}$.

Carrying on in this manner, we can define a decreasing sequence of subsets of $\Psi$ $\left\langle\Psi^{(n)}: n \in \omega\right\rangle$, and subtrees $T^{(n)}$ of $T$. It can be seen from above that if $\Psi^{(n)}$ is nonempty, then $T^{(n)}$ has elements at Level 2 , and so $\Psi^{(n+1)}$ is nonempty. But $\Psi$ is finite. Thus there is $n$ such that $\Psi^{(n)}=\Psi^{(n+1)}$. Put $\Phi=\Psi^{(n)}$. Let us assume that $n>1$ (w.n.l.o.g.). We first show transitivity.

So suppose that $M \vDash \bigvee \Phi(\bar{c}, \bar{d}) \wedge \bigvee \Phi(\bar{d}, \bar{e})$. Then $\bar{d}=\bar{d}_{i}$, and $\bar{e}=\bar{e}_{i, j}$, where it can be easily seen that $\bar{d}_{i}$ and $\bar{e}_{i, j}$ are in $T^{(n)}$. But $T^{(n)}$ is a subtree of $T^{(n-1)}$, and $\Phi=\Psi^{(n)}$ is just those $\psi$ such that for some $\bar{e}$ in Level 2 of $T^{(n-1)} M \vDash \psi(\bar{c}, \bar{e})$. So clearly $M \vDash \bigvee \Phi\left(\bar{c}, \bar{e}_{i, j}\right)$, and transitivity is proved.

Now we show denseness. First let $\psi \in \Phi$. So $\psi \in \Psi^{(n)}$ and thus also is in $\Psi^{(n+1)}$. But this means that there is $\bar{e}_{i, j}$ in $T^{(n)}$ such that $M \vDash \psi\left(\bar{c}, \bar{e}_{i, j}\right)$. Then $\bar{d}_{i}$ is in $T^{(n)}$, and so by the defintion of $T^{(n)}, M \vDash \vee \Psi^{(n)}\left(\bar{c}, \bar{d}_{i}\right) \wedge \bigvee \Psi^{(n)}\left(\bar{d}_{i}, \bar{e}_{i, j}\right)$. As $\psi(\bar{c}, \bar{x})$ is a complete formula of $\operatorname{Th}(M, \bar{c})$, it follows that

$$
M \vDash \forall \bar{x}(\psi(\bar{c}, \bar{x}) \rightarrow e \bar{y}(\bigvee \Phi(\bar{c}, \bar{y}) \wedge \bigvee \Phi(\bar{y}, \bar{x})))
$$

As this is true for every $\psi \in \Phi$, we have

$$
M \vDash \forall \bar{x}(\bigvee \Phi(\bar{c}, \bar{x}) \rightarrow E \bar{y}(\bigvee \Phi(\bar{c}, \bar{y}) \wedge \bigvee \Phi(\bar{y}, \bar{x})))
$$

Thus Lemma 8 is proved.

THeOREM 9. Let $T$ be a theory with an algebraic model, and few links. Then $T$ has infinitely many countable models.

Proof. Suppose that $T$ has an algebraic model and few links. We assume that $T$ has finitely many countable models and try to get a contradiction. The idea is to impose the dense order of Lemma 8 on the algebraic model, and to fabricate $2^{\boldsymbol{x}_{0}}$ types. So if $T$ has finitely many countable models, then as $T$ is clearly not $\aleph_{0}$-categorical (it has an algebraic model), we can work with Assumption 6. Therefore Lemma 8 holds. Let us write $\varphi(\bar{x}, \bar{y})$ for the formula $\bigvee \Phi(\bar{x}, \bar{y})$, where $\Phi$ is as in Lemma 8.

Let now $\sigma_{1}(\bar{c})$ be $\forall \bar{x} \bar{y}(\varphi(\bar{c}, \bar{x}) \wedge \varphi(\bar{x}, \bar{y}) \rightarrow \varphi(\bar{c}, \bar{y}))$. Let $\sigma_{2}(\bar{c})$ be $\forall \bar{x}(\varphi(\bar{c}, \bar{x}) \rightarrow$ $\operatorname{Ey}(\varphi(\bar{c}, \bar{y}) \wedge \varphi(\bar{y}, \bar{x})))$. Let $\sigma_{3}(\bar{c})$ be $\forall \bar{x}(\varphi(\bar{c}, \bar{x}) \rightarrow \bar{c} \neq \bar{x})$ and finally let $\sigma_{4}(\bar{c})$ be $E \bar{x} \varphi(\bar{c}, \bar{x})$.

Then by Lemma 8 and some remarks preceding it we have

$$
M \vDash \sigma_{1}(\bar{c}) \wedge \sigma_{2}(\bar{c}) \wedge \sigma_{3}(\bar{c}) \wedge \sigma_{4}(\bar{c}) .
$$

Thus as the type of $\bar{c}$ in $M$ is $p$, we have $T \cup p(z)+\bigwedge_{i=1, \ldots, 4} \sigma_{1}(\bar{z})$. So by compactness, there is $\chi_{1}$ in $p$ such that $T \cup\left\{\chi_{1}(\bar{z})\right\} \vdash \wedge_{i=1, \ldots, 4} \sigma_{i}(\bar{z})$. We also have $T \cup p(z) \vdash \varphi(\bar{z}, \bar{x}) \rightarrow p(\bar{x})$. Thus there is again $\chi_{2}(\bar{z})$ in $p$ such that $T \cup$ $\left\{\chi_{2}(\bar{z})\right\} \vdash \varphi(\bar{z}, \bar{x}) \rightarrow \chi_{1}(\bar{x})$. Let $\chi(z)$ be $\chi_{1}(\bar{z}) \wedge \chi_{2}(\bar{z})$. Thus 


$$
T \cup\{\chi(\bar{z})\} \vdash \bigwedge_{i=1, \ldots, 4} \sigma_{i}(\bar{z}) \wedge\left(\forall \bar{x}\left(\varphi(\bar{z}, \bar{x}) \rightarrow \bigwedge_{i=1, \ldots, 4} \sigma_{i}(\bar{x})\right)\right)
$$

Let now $N$ be the prime model of $T$ (which exists as we assume $T$ to have a finite number of countable models). Then as $T$ has an algebraic model, $N$ must be algebraic. Let us suppose that the type $p$ was an $n$-type, so all the tuples above are $n$-tuples. As $N$ is prime, every $n$-tuple of $N$ is in a complete $n$-formula. By the fact that $N$ is algebraic, any such complete $n$-formula must be finite (i.e. satisfied by only a finite number of $n$-tuples). From now on we will work inside the model $N$. By elements we will mean $n$-tuples. We will also identify formulae (always without parameters) and the sets of elements (tuples) they define in $N$.

First let $X=\{\bar{x} \in N: E \bar{z}(\chi(\bar{z} \wedge \varphi(\bar{z}, \bar{x})))\}$. Then by (*), $X$ is infinite. We will let $A_{i}, i \in \omega$, denote complete $n$-formulae such that $A_{i} \subseteq X$. If $A$ is complete and $A \cap X \neq \varnothing$, then $A \subseteq X$. For $A_{i}$ as above, we will use the following abbreviations (i) $A_{1}<A_{2}$ means $E \bar{x} \in A_{1} E \bar{y} \in A_{2}(\varphi(\bar{x}, \bar{y}))$, (ii) $\left(A_{1}, A_{2}\right)$ is the (definable) set $\{\bar{x}$ : $\left.E \bar{y} \in A_{1} E \bar{w} \in A_{2}(\varphi(\bar{y}, \bar{x}) \wedge \varphi(\bar{x}, \bar{w}))\right\}$.

We now make a series of remarks.

Remark 10. Suppose that $A_{1}<A_{2}$. Then $\left(A_{1}, A_{2}\right)$ is a nonempty subset of $X$.

Proof. Let $\bar{a} \in A_{1}$, and $\bar{b} \in A_{2}$, such that $\varphi(\bar{a}, \bar{b})$. Then as $A_{1} \subseteq X$, we have by (*) that $\sigma_{2}(\bar{a})$. Thus there is $\bar{c}$ such that $\varphi(\bar{a}, \bar{c})$ and $\varphi(\bar{c}, \bar{b})$. So $\left(A_{1}, A_{2}\right)$ is nonempty. On the other hand, let $\bar{d} \in \chi^{N}$, such that $\varphi(\bar{d}, \bar{a})$ and suppose $\varphi(\bar{a}, \bar{c})$. Then by (*), we have $\sigma_{1}(\bar{d})$, and so $\varphi(\bar{d}, \bar{c})$, whereby $\bar{c} \in X$. Thus $\left(A_{1}, A_{2}\right)$ is a subset of $X$.

Remark 11. Suppose that $A_{1}<A_{2}$. Then there is $A_{3}$ such that $A_{1}<A_{3}$ and $A_{3}<A_{2}$.

Proof. This is immediate from the previous remark, as the definable set $\left(A_{1}, A_{2}\right)$ must contain a complete formula.

Remark 12. For any $A_{i}$, it is not the case that $A_{i}<A_{i}$.

Proof. Suppose that we had $A_{i}<A_{i}$. Then there would be $\bar{a}, \bar{b}$ in $A_{i}$ such that $\varphi(\bar{a}, \bar{b})$. Thus as $A_{i}$ is finite, and $\varphi$ is transitive and irreflexive on $A_{\underline{i}}$ (by (*)), there is $\bar{a}, \bar{b}$ in $A_{i}$ such that $\varphi(\bar{a}, \bar{b})$, and there is no $\bar{x}$ in $A_{i}$ such that $\varphi(\bar{b}, \bar{x})$. But as $A_{i}$ is complete, $\bar{a}$ and $\bar{b}$ have the same type, whereby there is no $\bar{x}$ in $A_{i}$ such that $\varphi(\bar{a}, \bar{x})$. This is clearly a contradiction. So the remark is proved.

Remark 13. Suppose that $A_{1}<A_{3}$ and $A_{3}<A_{2}$. Then $\left(A_{1}, A_{3}\right) \cap\left(A_{3}, A_{2}\right)=\varnothing$.

Proof. If not then there is $\bar{a}$ in $\left(A_{1}, A_{3}\right)$ and $\left(A_{3}, A_{2}\right)$. So there are $\bar{b}, \bar{c}$ in $A_{3}$ such that $\varphi(\bar{a}, \bar{b})$ and $\varphi(\bar{c}, \bar{a})$. As $A_{3} \subseteq X$, we have $\sigma_{1}(\bar{c})$ and so $\varphi(\bar{c}, \bar{b})$. But this contradicts Remark 12.

Now as $X$ is infinite and by (*) we can easily find a nonempty interval of the form $\left(A_{1}, A_{2}\right)$ contained in $X$. By the above remarks, any such nonempty interval can be divided into two disjoint nonempty intervals in $X$ of the same form. All these intervals are definable subsets.

Thus for each $\tau \in 2^{<\omega}$, we can define a definable set $X_{\tau}$ in $N$, such that each $X_{\tau}$ is nonempty,

$$
X_{\tau \cap\langle 0\rangle} \cup X_{\tau \cap\langle 1\rangle} \subseteq X_{\tau} \text { and } X_{\tau \cap\langle 0\rangle} \cap X_{\tau \cap\langle 1\rangle}=\varnothing \text {, }
$$


for all $\tau \in 2^{<\omega}$. For each $\eta \in 2^{\omega},\left\{X_{\eta \mid i}: i<\omega\right\}$ is a consistent set of formulae. Thus we clearly get $2^{\kappa_{0}} n$-types of $T$. But then $T$ must have $2^{k_{0}}$ countable models, which contradicts our assumption that it had finitely many. This contradiction proves Theorem 9.

\section{REFERENCES}

1. M. Benda, Remarks on countable models, Fund. Math. 81 (1974), 107-119.

2. S. Shelah, Stability, the f. c. p. and superstability, Ann. Math. Logic 3 (1971), 271-362.

3. A. Pillay, Theories with exactly three models, and theories with algebraic prime models, J. Symbolic Logic (to appear).

Universite de Paris Vil, 2 Place Jussieu, 75221 Paris Cebdex 05, Franct

Current address: 73 Twyford Avenue, London N.2 9NP, England 